\title{
Romans historiques pour la jeunesse et construction de savoirs scolaires en histoire (cycle 3)
}

Historical Fictions for Youth and Construction of School Knowledge in History (Elementary School)

Sylvie Lalagüe-Dulac

\section{OpenEdition} Journals

Édition électronique

URL : https://journals.openedition.org/educationdidactique/2685

DOI : $10.4000 /$ educationdidactique.2685

ISSN : 2111-4838

\section{Éditeur}

Presses universitaires de Rennes

\section{Édition imprimée}

Date de publication : 20 juin 2017

Pagination : 105-121

ISBN : 978-2-7535-6460-2

ISSN : $1956-3485$

\section{Référence électronique}

Sylvie Lalagüe-Dulac, «Romans historiques pour la jeunesse et construction de savoirs scolaires en histoire (cycle 3) », Éducation et didactique [En ligne], 11-1 | 2017, mis en ligne le 20 juin 2019, consulté le 14 septembre 2022. URL : http://journals.openedition.org/educationdidactique/2685 ; DOl : https:// doi.org/10.4000/educationdidactique.2685 


\title{
ROMANS HISTORIQUES POUR LA JEUNESSE ET CONSTRUCTION DE SAVOIRS SCOLAIRES EN HISTOIRE (CYCLE 3)
}

\author{
Sylvie Lalagüe-Dulac \\ Maître de conférences en didactique de l'histoire à l'université de Bordeaux, ESPE \\ d'Aquitaine. Laboratoire Lab-E3D, université de Bordeaux.
}

\begin{abstract}
Lutilisation de fictions historiques séduit régulièrement nombre d'enseignants du primaire qui, encouragés par les prescriptions officielles, choisissent d'étudier en histoire des œuvres susceptibles de captiver leurs élèves et de leur faire prendre conscience du passé, car, à leurs yeux, le roman historique éclaire l'histoire. Or, l'élève fait-il la distinction entre la fiction et la réalité dont la dichotomie interroge les frontières entre les disciplines d'histoire et de français et la polyvalence des maîtres ? Que se passe-t-il quand un élève lit un roman historique dans une perspective de savoir partagé ? Que vont apporter ses souvenirs de lecture à sa compréhension des événements historiques?
\end{abstract}

Mots-clés : histoire, fiction, élève-lecteur, conception, polyvalence.

\section{Historical Fictions for Youth and Construction of School Knowledge in History (Elementary School)}

The use of historical fiction regularly attracts many primary school teachers who, encouraged by the French official instructions, choose to study in history lessons some works likely to capture their pupils'attention and to make them become aware of the past, because, in their eyes, historicals novels explain history. However, does the pupil distinguish between fiction and reality whose dichotomy questions the borders between the disciplines of history and French and the polyvalence of the teachers? What occurs when a pupil reads a historical novel so as to share knowledge? What will his/her memories of reading bring to his/her comprehension of the historical events?

Keywords: history, fiction, pupil-reader, conception, polyvalence. 


\section{INTRODUCTION}

L'objet de cet article est de questionner l'utilisation de romans historiques pour la jeunesse en classe d'histoire et sa pertinence dans la construction de savoirs historiques scolaires partagés au cycle $3^{1}$. Né au XIX ${ }^{e}$ siècle dans un contexte propre à son éclosion, le roman historique est devenu un genre romanesque à part entière (Manson, 2013). Eric Bordas (2002, p. 171) le définit « comme un récit dont le cadre chronologique renvoie à des situations et des faits bien connus de l'histoire politique des hommes, proche ou ancienne, un récit dont plusieurs personnages, principaux ou secondaires, sont des figures attestées et notoires de la mémoire d'un pays ». La fiction se doit d'y être vraisemblable et d'éviter tout élément anachronique bien que l'achronie soit souvent de règle, flou chronologique aux multiples avantages pour l'écrivain (Baguley, 2008, p. 78 ; Bessière \& Sinoploi, 2005). André Peyronie (2000, p. 281) insiste, quant à lui, sur « la nécessaire distance temporelle entre l'époque concernée et l'écriture du roman ». Le résultat est un véritable exercice de style nécessitant une grande habileté car le roman historique est avant tout un genre littéraire qui doit séduire le lecteur inconscient de la frontière presque invisible entre le réel et l'imaginaire (Peyronie, 2000, p. 289).

Rédigé d'abord à l'intention d'adultes, il s'est rapidement adressé à la jeunesse. Tout en mettant en scène des faits historiques attestés et en dressant un tableau de la société concernée le plus fidèle possible, le roman historique pour la jeunesse suit le destin individuel d'un enfant ou d'un adolescent, souvent un garçon, confronté aux vicissitudes de l'histoire dont il sort généralement vainqueur (Bruno \& Geneste, 2008, p. 417-423). Son succès (Gengembre, 2006, p. 123-124) s'est accéléré auprès des jeunes lecteurs ces dernières décennies autour de différentes catégories $^{2}$ : le roman d'aventures, le roman de société, le roman policier... Dans tous les cas de figure, l'histoire, essentiellement nationale, sert de cadre aux fictions proposées. Ce cadre n'étant pas forcément connu du lecteur ni même de l'auteur, ce dernier a l'obligation, dans un premier temps, de se documenter, de mener sa propre enquête ${ }^{3}$ afin de pouvoir, dans un second temps, apporter, dans sa reconstitution, les explications nécessaires à la bonne compréhension du contexte dans lequel il place son roman.
La subtile relation qui lie histoire et roman historique pour la jeunesse sera examinée en lien avec la didactique de l'histoire et ses finalités. Que se passet-il lorsque des élèves étudient une question historique en s'appuyant sur la lecture totale ou partielle d'un roman historique pour la jeunesse? Adoptentils une posture critique ou sont-ils plongés dans l'empathie la plus totale ? Quelles interprétations opèrent-ils et sont-elles convergentes ? Si la lecture s'est effectuée en amont en cours de littérature, que vont apporter leurs souvenirs de lecture à leur compréhension des événements historiques étudiés en classe d'histoire et ces souvenirs vont-ils interférer avec les savoirs historiques en construction ? La lecture d'un roman historique ne pouvant conduire à la mémorisation classique - il ne peut être appris par cœur - qu'en retiennent les élèves ?

\section{LE POIDS DES REPRÉSENTATIONS}

Didactique de l'histoire ${ }^{4}$ et didactique de la littérature prennent chacune en compte les représentations mentales mais avec des finalités et des conséquences différentes.

\section{De l'analogie aux représentations en histoire}

Pour penser en histoire (Lautier, 2001 ; Lautier \& Allieu-Mary, 2008), l'élève doit tenter de procéder à la mise en ouvre de procédés d'historisation nécessaires à la formalisation et à la rationalisation du savoir scolaire, soit :

- la critique des sources ;

- le contrôle du raisonnement comparatif ;

- la périodisation pour construire le temps historique différent du temps subjectif ;

- le contrôle du degré de généralisation des concepts ;

- et l'élaboration des entités, ces « quasi-personnages » de l'histoire.

L'étude de documents de diverses natures est à la base du travail de l'élève comme elle l'est pour l'historien car l'histoire est une connaissance par traces qu'il faut sans cesse questionner ainsi que le rappelait Henri Moniot (1993, p. 49) : «l'histoire se fait avec des restes - on préfère dire, de façon plus distinguée: avec des documents ou avec des sources $»$. 
Reconstituer et comprendre l'évolution de l'humanité dans le temps suppose également la capacité à prendre en considération l'univers mental des acteurs de l'histoire. En effet, cette discipline met constamment l'élève en relation avec un « Autre » plus ou moins lointain (Heimberg, 2005), plus ou moins passé et le raisonnement par analogie est essentiel pour faire de l'histoire (Cariou, 2003a; Deleplace, 2006). Les hommes étant les seuls objets d'étude de l'histoire, «l'historien raisonne par analogie avec le présent, il transfère au passé des modes d'explication qui ont fait leur preuve dans l'expérience quotidienne de tout un chacun » (Prost, 1996, p. 159) et, du point de vue de la logique, l'explication de l'historien ne diffèrerait pas de celle du non-spécialiste. Aussi, « aucune compétence spécifique n'est-elle requise du lecteur pour pénétrer dans un livre d'histoire » (id., 1996, p. 159), si ce n'est d'avoir de l'expérience, du « vécu ». L'élève ne procèderait pas autrement. Lorsqu'il lui est demandé une analyse historique, il mobiliserait également sa connaissance du monde vécu (Moniot, 1993, p. 123).

Deux modalités du raisonnement analogique ont cependant été distinguées chez les élèves (Lautier, 1997, p. 89-91 ; Cariou, 2003b). L'analogie passéprésent qui permet la compréhension du passé par analogie au présent ou par voisinage et l'analogie passé-passé, plus complexe, qui nécessite une culture historique préexistante. Les élèves interprèteraient de ce fait l'histoire à l'aide de leur monde " par le biais d'un jugement naif qui relève également du processus de la figuration des représentations sociales » (Cariou, 2004, p. 62).

Les représentations ${ }^{5}$ sociales $^{6}$ sont construites autour de schémas cognitifs de base et « ancrées au sein d'un groupe et du système de valeurs qui lui est propre, [...] toute information nouvelle étant interprétée dans les cadres mentaux préexistants» (Dortier, 2004, p. 634). Modèle explicatif, les conceptions (le terme a désormais remplacé celui de "représentations $»^{7}$ ) ont une genèse à la fois individuelle et sociale car elles sont « un processus personnel, par lequel l'apprenant structure au fur et à mesure les connaissances qu'il intègre [...] à partir de l'action culturelle parentale, de sa pratique sociale d'enfant à l'école, des divers médias et, plus tard, de son activité professionnelle et sociale d'adultes » (Gordian et de Vecchi, 1987, p. 85). Ce « savoir initial », fait d'idées et de concepts, est une sorte de crible au travers duquel les apprenants essaient de comprendre les propos de l'enseignant ou les documents étudiés, mais il est toujours actualisé par la situation vécue, par les questions posées, à savoir le contexte . $^{8}$

Confrontées à une nouvelle situation, " tout à la fois, les conceptions antérieures filtrent, trient et élaborent les informations reçues et, en retour, peuvent être parfois complétées, limitées ou transformées » (id., p. 86), tout en donnant naissance à de nouvelles conceptions, ce qui permet à l'apprenant de constituer une grille de lecture applicable à son environnement. Par le biais de cet outil d'analyse, l'élève peut « organiser le monde, ou un aspect du monde, de telle manière qu'il puisse le comprendre, agir sur lui, s'y adapter ou s'en évader, du moins à un certain niveau » (id., p. 86). Or, le terme de « conceptions » renvoyant à l'ensemble de l'univers conceptuel - le déjà là - d'un individu (Clément, 1994, p. 20), il paraît logique de supposer que les conceptions produites par chacun sont très variées et « originales». De plus, pendant un apprentissage, ce qu'exprime un élève " ne traduit pas directement l'ensemble de ses conceptions, mais seulement ses conceptions structurelles : celles qui sont mobilisées (en mémoire de travail) dans la situation précise » (id., p. 21) et qu'il extrait de sa mémoire permanente.

\section{Représentations et roman historique pour la jeunesse}

La lecture de roman historique pour la jeunesse favorisant l'imaginaire, la fictionnalisation, on peut supposer que, pour certains, les conceptions générées, ou peut-être déjà présentes, mais remodelées par une lecture en séance d'histoire, ne correspondront pas nécessairement à l'objectif assigné par l'enseignant dans ce cadre, à savoir la construction d'un savoir scolaire historique partagé reposant sur des faits politiques, économiques, sociaux ou culturels contextualisés et authentiques. D'autant plus que les structures mentales humaines ne seraient pas spontanément organisées en structures distinctes séparant ce qui est scientifiquement attesté, validé, de ce qui ne l'est pas. Nos connaissances formeraient « un tout où se mêlent connaissances scientifiques, croyances, idéologies, fonctionnalités sociales, dimensions rationnelles et esthétiques, émotionnelles, affectives. Ces conceptions sont en permanence confortées par nos pratiques humaines donc sociales » (Clément, 1994, p. 28). 
Or, si, pour les spécialistes de la littérature, « les troubles, les émotions, les rêveries, les associations d'idées, voire les rapprochements impromptus, qui puisent leurs racines dans la personnalité profonde, l'histoire personnelle, les souvenirs littéraires ou d'instants de vie de l'individu qui lit» peuvent être considérés « comme des éléments parasites qui faussent, brouillent et embrouillent la réception d'une œuvre » (Langlade, 2004, p. 81) puisque nous sommes avant tout sensibles lors de la lecture d'un roman à ce qui nous concerne nous-mêmes ${ }^{9}$, que dire de cette réception du point de vue de l'histoire? Le texte vivant de ses retentissements avec ses souvenirs, ses images mentales, ses représentations intimes de lui, des autres, de son monde, comment l'élève peut-il adopter la distance du lecteur critique selon le modèle de l'historien ? Il ne peut être que partagé entre des questions précises visant à faire émerger des faits, des caractéristiques, des liens de causalité en relation avec des savoirs et ce que le texte lui renvoie de son expérience du monde, de ses souvenirs personnels, de son histoire propre. Et la perception des événements du passé sera de moins en moins historique et de plus en plus romanesque (Ansel, 2000, p. 113) et singulière.

\section{Personnages : qui est qui ?}

La nécessité propre à l'histoire de développer l'esprit critique des élèves pose un autre problème quant à l'usage de fiction historique en séance d'histoire, celui du rapport des élèves aux personnages, rapport qui soulève deux questions qui s'entrelacent, celles de leur historicité et celle du processus d'identification régulièrement constaté.

Dans le roman historique, les personnages principaux sont généralement fictifs ${ }^{10}$, bien que ce ne soit pas toujours le cas. Ainsi, d'Artagnan et le cardinal de Richelieu, dans les Trois mousquetaires, sont des héros bien réels, qui côtoient des personnages fictifs comme les Bonacieux, Milady ou Lord de Winter.

La perception de la réalité relevant de celle du statut des personnages, comment, cependant, discerner les personnages référentiels, " historiques ", ancrant la fiction dans la réalité, des personnages créés de toutes pièces pour les besoins de la fiction, les premiers accréditant les seconds ? Difficulté amplifiée par le fait que les caractéristiques du héros fictif doivent correspondre à la réalité histo- rique de l'époque choisie par souci de vraisemblance historique.

La classification proposée par André Peyronie (2000, p. 284-287) dans son analyse du roman, Le Nom de la rose, met en valeur la sophistication de cet artifice. Il a établi une échelle d'historicité dans laquelle s'inscrivent les différents personnages. Le premier groupe rassemble des personnages historiques qui ont vécu en un lieu lointain ou à une autre époque (Aristote, Thomas d'Aquin...), ce sont les « historiques-mentionnés ». Le second groupe réunit des personnages historiques contemporains qui ont pu jouer un rôle dans l'action sans être présents à l'abbaye (le pape, l'empereur...), ils sont dits « historiques-rattachés ». Un troisième groupe est constitué de contemporains ayant véritablement existé et qui apparaissent à l'abbaye (Urbain de Casale, Bernard Gui...), ce seraient les « historiques-actants ». La quatrième catégorie renvoie aux personnages romanesques, inventés et présents dans l'action mais qui peuvent avoir des modèles dans la réalité (Adso, les moines...), ils appartiendraient à la catégorie des « fictifs-actants». Enfin, une cinquième catégorie pourrait correspondre aux personnages inventés mais qui n'apparaissent pas " physiquement » dans le roman comme les parents d'Adso, ce seraient des « fictifs-rattachés ».

Cet effort de catégorisation traduit la complexité dont la littérature se nourrit, mais cette même complexité peut représenter pour l'enseignement de l'histoire un obstacle en raison de la subtilité requise de la part de jeunes lecteurs. Ainsi, les dialogues entre les personnages sont "imaginés » et engagent l'élève davantage dans la subjectivité du romancier que dans la stricte réalité historique. Les conséquences, en termes de construction de savoirs scolaires, en sont peut-être plus importantes lorsqu'il s'agit des grands hommes de l'histoire dont les propos ne pourront jamais être véritablement inscrits dans « le registre de leurs voix et de leurs âmes ${ }^{11}$ ", mais ils seront cependant inscrits en tant que tels dans la mémoire des élèves. De fait, même lorsque les personnages ont un référent réel, « ils restent des entités romanesques, des êtres de papier, comme les décors sont des architectures des mots $»($ Peyronie, 2000, p. 279).

Autre point important, la fiction étant généralement livrée linéairement ou chronologiquement, l'histoire individuelle est privilégiée à l'histoire collective et provoque un effet d'identification du lecteur avec le personnage principal (Bruno \& 
Geneste, 2008, p. 417-423) d'autant que, comme le souligne Michel Manson (2013, p. 822), les auteurs dotent « les personnages d'enfants d'une psychologie contemporaine pour que le lecteur puisse s'identifier $»$. Brigitte Louichon (2014, p. 174) rappelle également que « l'auteur contemporain de roman historique écrit pour un lecteur contemporain une histoire se passant autrefois ». Il n'hésite pas à user d'anachronisme langagier privilégiant le lecteur et la fiction aux dépens de l'histoire, anachronisme qui permet aux élèves de ne pas sentir toute la densité de l'épaisseur du temps passé et, de ce fait, de l'en rapprocher, les plaçant dans un espace-temps incertain.

Les nombreux blancs présents dans les textes littéraires sont aussi autant de tentation pour chaque élève de manifester sa singularité en cherchant consciemment ou inconsciemment à compléter, à partir de détails minuscules, des pans entiers de la vie d'un personnage ${ }^{12}$, part de subjectivité considérée par beaucoup comme une réalité négative, " l'implication personnelle du lecteur dans le texte contenant en germe toutes les déviances possibles, de la simple erreur de lecture au contresens le plus grave » (Jouve, 2008, p. 105). La fonction implicative $^{13}$, décrite par Yves Reuter, puisque le récit a pour objet d'engager l'élève dans le travail, peut avoir cet effet négatif de le «sortir de sa position d'extériorité, soit en jouant sur l'articulation cognition-affects, soit en jouant sur l'implication via les personnages, sur le désir de connaître la suite ou d'en connaître plus ; soit en construisant des modes de contextualisation des contenus censés renvoyés au vécu et à la culture des élèves » (Reuter, 2007, p. 10). Or c'est cette position d'extériorité ${ }^{14}$ qui est nécessaire à la démarche historienne. Si elle disparaît, la frontière entre la réalité et la fiction est encore plus difficile à percevoir.

\section{Lélève lecteur}

Lire durant une séance d'histoire un roman historique pour la jeunesse conduit à s'intéresser à l'élève lecteur appelé, depuis les travaux de Michel Picard, « lecteur réel » (1986). Lire un document écrit, quel qu'il soit, en histoire répond à une démarche, en principe bien connue des élèves de cycle 3 , calquée sur les pratiques des historiens. Si nous schématisons rapidement, cela consiste, dans un premier temps, à identifier formellement la nature, la date et l'auteur, donc à authentifier le document en question, puis, dans un second temps, à hiérarchiser les informations contenues dans ce document afin de produire, à partir d'un questionnement écrit ou oral, un texte informatif et explicatif permettant de comprendre le fait historique étudié. Des résultats des recherches menées par les spécialistes de littérature et de sa didactique, il ressort que la lecture dans une situation d'enseignement du point de vue $\mathrm{du}$ français est un acte bien plus complexe que pourrait le supposer un béotien en la matière. Les raisons, présentées sans aucune prétention à l'exhaustivité, sont multiples. L'élève relèverait de la catégorie du lecteur réel, un lecteur « ordinaire», « naif » ou « faible » (Dufays, 2013). Or, un lecteur doit réussir « à "prendre ensemble" (cf. l'étymologie latine comprendere) toutes les données du texte et les relier entre elles en un tout cohérent . »(Goigoux \& Cèbe, 2011, p. 35-36). Pour saisir la représentation mentale de l'ensemble de la situation exposée dans un texte littéraire, " le lecteur doit aller au-delà de ce que le texte dit explicitement et procéder à des inférences temporelles, sur l'ordre des actions, et des inférences causales» (id., p. 36). Or, l'exploitation, en classe d'histoire de romans historiques pour la jeunesse s'inscrivant dans des périodes historiques précises se heurte à la culture historique généralement très faible des élèves de cycle 3 dont les compétences encyclopédiques sont limitées.

En outre, le modèle mental élaboré à partir du texte du lecteur ${ }^{15}$ élève ne correspondrait pas au modèle mental construit par le professeur : «le "texte du lecteur-maitre", produit d'une lecture en principe plus outillée et plus orientée que celles des apprenants, diffère des "textes des lecteurs-élèves", qui diffèrent eux-mêmes peu ou prou les uns des autres. » (Dumortier, Dispy \& Van Beveren, 2011, p. 32). Tout lecteur aurait la particularité de conjuguer plusieurs identités lorsqu'il lit...

Alors, les interprétations des élèves, renvoyés par chaque phrase à leurs conceptions propres, à leurs souvenirs et confrontés à la dichotomie fiction / réalité et au raisonnement par analogie, peuvent-elles être correctes et cohérentes avec le savoir historique visé ? Si l'assertion longtemps utilisée en biologie à savoir " il n'y a qu'à regarder pour comprendre » a pu être déconstruite, est-il légitime de continuer à penser qu'il n'y a qu'à « lire pour comprendre »? Car tout regard étant, indissociablement, interpré- 
tation sur ce qui est vu, ce qui ne peut être interprété n'est en général pas vu (Clément, 1994, p. 35), aussi que « voient » les élèves lors de la lecture d'un roman historique? Comment réussissent-ils à faire entendre dans une séance d'histoire, où l'objectif est de construire des savoirs partagés par tous dans une visée de culture humaniste, tant de lectures différentes, "cacophonie " intérieure ou affichée qui se heurte en outre à un autre obstacle en lien avec tous ceux qui viennent d'être exposés.

\section{Deux communautés discursives disciplinaires singulières}

Exploiter une fiction historique pour la jeunesse en classe d'histoire, c'est faire cohabiter deux Communautés Discursives Disciplinaires Scolaires (Bernié, 2002 ; Bernié, Jaubert \& Rebière, 2004) que nous appellerons «Communauté Discursive Littéraire Scolaire », soit CDLS, pour la littérature et "Communauté Discursive Historienne Scolaire ", soit CDHS, pour l'histoire. Une Communauté Discursive Disciplinaire Scolaire ne peut se construire que par référence en actes aux pratiques sociales et langagières des communautés de référence. L'élève doit, par un positionnement énonciatif particulier dans un champ d'activité humaine donné, se constituer en "sujet scientifique scolaire " tout en secondarisant ses pratiques langagières. Il doit s'instituer acteur dans une communauté transposée à l'école en s'appropriant ses pratiques à la fois technologiques et langagières (Bernié, 2002, p. 82-83). Exploiter un roman historique en classe d'histoire peut perturber certains élèves ne sachant pas dans quelle Communauté Discursive Disciplinaire ils doivent s'inscrire. Doivent-ils adhérer à une CDLS ou à une CDHS ? Quel positionnement énonciatif doivent-ils adopter, doivent-ils « lire » le texte selon les pratiques des historiens ou selon celles apprises face à un texte littéraire, ces pratiques n'étant pas du tout les mêmes qu'ils participent à un cours d'histoire ou de littérature? Ainsi, pour que la construction d'une Communauté Discursive Historienne Scolaire s'opère, il faut que se mettent en place les pratiques matérielles et langagières transposées de la communauté des historiens : critique des traces laissées par les hommes du passé, analyse, interprétation, raisonnement par analogie, périodisation et généralisation. Pratiques qui favorisent la construction d'un récit qui se veut le plus historique possible car validé par ces procédures.

De ce fait, les élèves doivent, au cours d'une séance d'histoire, adopter une position énonciative et des pratiques langagières et matérielles qui donnent à voir celle de la communauté des historiens, mais dans quelle communauté de recherche la lecture d'un roman historique pour la jeunesse favorise-telle l'inscription des élèves?

Pour qu'une CDLS s'instaure en classe de littérature, on peut penser avec Bernié et al. (2004) que les pratiques qui se mettent en place le sont en référence aux manières de penser, de parler, d'agir de la communauté lettrée des écrivains ou des critiques littéraires, l'école devant conduire les élèves à la lecture distanciée du lettré tout en ne négligeant pas les apports de la lecture subjective. L'identification et l'illusion référentielle relevant de l'expérience littéraire (Vibert, 2013, p. 12) seraient grandement préférables à la posture d'extériorité qui, du côté des historiens, est considérée comme essentielle... Les pratiques discursives, les positionnements énonciatifs et les savoirs visés sont donc nécessairement distincts de ceux attendus en classe d'histoire. L'exploitation d'un même objet culturel, le roman historique, par ces deux didactiques dont les cadres théoriques et les finalités sont différents, voire difficilement compatibles, peut-elle être cependant profitable à la construction de connaissances spécifiques autour de ce même contenu ? La fiction historique permet-elle aux élèves de saisir les modifications de l'intervention des agents sur le cours de l'histoire (Cariou, 2006 ; Doussot, 2011) davantage que les pratiques dominantes de classe d'histoire? La faiblesse des activités sollicitées par les pratiques ordinaires en classe d'histoire, qualifiées de «paradigme pédagogique positiviste » (Tutiaux-Guillon, 2009, p. 109), est en effet régulièrement soulignée par les didacticiens de l'histoire. Le cours magistral dialogué voit sans fin se répéter des boucles didactiques telles que Nicole Tutiaux-Guillon (1998; 2009) a pu les mettre en évidence, pratiques qui tendent souvent à réduire le savoir au vrai et donc à faire rechercher la bonne réponse dans des documents fournis par l'enseignant (Doussot, 2011, p. 26). 


\section{UTILISER DES ROMANS HISTORIQUES EN CLASSE D'HISTOIRE : QUELQUES EXEMPLES}

Qu'ils soient encouragés par le discours officiel ou qu'ils le pratiquent déjà depuis longtemps, j’ai pu constater grâce aux réponses à des questionnaires soumis à cent sept enseignants du primaire qu'en Gironde, nombre d'enseignants choisissent, assez fréquemment, d'étudier en histoire des fictions historiques pour deux raisons principales. D'une part, elles seraient susceptibles de captiver leurs élèves et de leur faire prendre conscience du passé rejoignant ainsi les conclusions d'Umberto Eco pour lequel les agissements des personnages serviraient « à mieux faire comprendre l'histoire, ce qui s'est passé, et bien qu'ils soient inventés, ils en disent plus, et avec une clarté sans pareille, sur [...] l'époque, que les livres d'histoire consacrés» (Eco, 1985, p. 87). D'autre part, varier les modalités pédagogiques, surprendre, étant d'un intérêt vital à l'école, le roman historique offrirait une bouffée d'air frais bienvenue au milieu d'une programmation serrée en jouant sur la pluridisciplinarité et la polyvalence. Nombre de ces réponses rejoignent la richesse des fonctions didactiques attribuées au récit par Yves Reuter (2007, p. 9-10). Au nombre de sept, trois d'entre elles ${ }^{16}$ semblent plus particulièrement en adéquation avec l'utilisation du roman historique en histoire. La fonction objectivante du récit permet d'actualiser, d'incarner ce qui est absent. Sa fonction médiatrice sert de palier pour progresser, pour susciter la réflexivité. Enfin, sa fonction implicative engage l'élève dans le travail et le sort de sa position d'extériorité.

Les exemples présentés s'appuient sur des recherches menées tout au long de ces dernières années dans divers contextes. De l'ensemble du corpus rassemblée ${ }^{17}$, seront plus particulièrement exploités quelques extraits de transcriptions de séances menées en classe d'histoire en CMl au cycle 3 en Gironde, séances reposant sur l'étude de romans historiques. Les quatre professeurs des écoles concernés, tous titulaires et expérimentés, ont utilisé, chacun, une des fictions suivantes : le roman de Jacqueline Mirande, Sans nom, ni blason, celui d'Evelyne Brisou-Pellen, Deux graines de cacao et celui de Jean-Côme Noguès, Le faucon déniché, dans l'intention, pour trois d'entre eux, de favoriser ou de consolider la construction de savoirs historiques scolaires et, pour l'un d'entre eux qui avait choisi d'étudier Deux graines de cacao, d'éduquer également ses élèves aux droits de l'Homme ${ }^{18}$. Les situations de classe présentées relèvent toutes de pratiques ordinaires, exceptée pour l'une d'entre elle qui a été, en partie, accompagnée de questionnaires soumis successivement à des élèves ayant lu, en classe, tout ou partie du roman de Jean-Côme Noguès, Le faucon déniché. Ces questionnaires ${ }^{19}$ ont été construits dans l'intention d'analyser plus finement les représentations des élèves de cette classe en fonction des chapitres lus en classe.

\section{Premiers obstacles}

Un professeur d'école a décidé d'illustrer, aprèscoup, la leçon sur les relations seigneurs / paysans à l'aide de la lecture d'extraits du roman Sans nom, ni blason de Jacqueline Mirande afin de confronter fiction et réalité. L'objectif de la première séance était de repérer des informations dans le texte littéraire et de confronter les données du roman à la réalité historique. Il avait prévu un va-et-vient constant du français à l'histoire et inversement. Les élèves, après avoir compris l'action, étaient priés de revenir au contexte historique par le biais de questions précises, de fiches documentaires photocopiées ou présentes dans leur manuel afin de visualiser correctement les lieux de l'intrigue et d'en comprendre plus finement le déroulement.

Sa première consigne illustre d'emblée la complexité de la tâche dans laquelle il lance ses élèves :

«1. M. : Bon alors je vais vous dire comment, je vais vous dire comment on va fonctionner. Vous avez une lecture, attends avant de la prendre, je vais vous donner XX les documents qui vont avec et quand quelque chose que vous ne comprenez, pas vous aurez les renseignements là-dessus d'accord ? [...] Et --- et, une feuille, celle-là, qui sert de document et il y a aussi des exercices, y a des choses à comprendre, c'est un peu un mélange de tout, je distribue des photocopies...»

Les élèves reçoivent alors les premières pages photocopiées du premier chapitre du roman mais, également, des fiches documentaires dans lesquelles ils trouvent du vocabulaire et un plan de la région qui devraient les aider à mieux comprendre le roman. Mais le maître termine en précisant dans cette première intervention «c'est un peu un 
mélange de tout ». L'expression « mélange de tout» résume ce qui va suivre. Durant toute la séance, l'enseignant passe d'une communauté discursive à l'autre en permanence en utilisant un vocabulaire, des consignes et des postures propres à l'une et à l'autre. « Livre », « chapitre », « texte», « qu'estce qui se passe ? ", " bruit mystérieux », " grand mince »... alternent avec « documents », « lexique», « définition », « vocabulaire », " renseignement ", « connaissances ».

Conscient des difficultés de l'exercice, en particulier sur le plan lexical, il dit, dès la première minute, en 11, « Il vous manquera des renseignements que vous prendrez sur votre livre ", puis six tours de parole plus loin « Bon, vous vous rappelez le livre dont je vous ai parlé on va prendre le chapitre 1 », mais l'élève fait-il la distinction entre le livre d'histoire et le livre à lire ? Peut-il être, dans ce contexte particulier, sensible à la part d'imagination, d'invention spécifique à la dimension littéraire de l'œuvre contenue dans un roman historique? D'autant que soucieux d'ancrer la lecture de Sans nom ni blason dans un contexte historique précis et « authentique », le professeur n'hésite pas, après la lecture d'un autre passage du roman, à demander aux élèves de réinvestir leurs connaissances historiques afin qu'ils comprennent les conséquences de la fuite de Guillaume.
Les élèves mettent six minutes pour trouver les phrases du roman concerné : «Tu ne connais donc pas l'obligation faite à tout serf de demeurer sur la terre de son seigneur sans la quitter jamais ni de jour ni de nuit » (Mirande, 1997, p. 7) et «Tu es lié à lui à nous autant qu'à sa terre et tu lui dois obéissance » (id., p. 8). S'ensuit un long échange sur les droits et les devoirs des serfs, puis le maître décide de revenir à l'approche littéraire du roman par un questionnement qui a pour ambition de replacer les élèves dans une CDLS : « Mais qu'est-ce qui se passe là ? » pour en arriver, enfin, à la question que certains élèves ne se posent plus : " Pourquoi arrive-t-il à cette abbaye, ce Guillaume ? Comment est-il ? ». Il tente ainsi de souligner un fait saillant afin de permettre une mise en intrigue et la réponse des élèves - « blessé », « il saigne »- les replonge effectivement jusqu'à la fin de la séance dans l'intrigue qui avait commencé à susciter leur intérêt un petit plus tôt et sur laquelle nous allons revenir un peu plus loin.

Très vite cependant, une autre difficulté pointée par Claudine Garcia-Debanc (1991) surgit : celle de la gestion du vocabulaire spécifique et des concepts nouveaux, moyen privilégié par le romancier pour créer l'atmosphère ad hoc. Relater des faits passés conduit l'auteur par souci de vraisemblance à employer, avec précision, nombre de termes tech-

\begin{tabular}{|c|c|c|}
\hline 475 & M. & $\begin{array}{l}\text { Bon, stop. Non, non, tu écoutes. Dans ce morceau, dans ce morceau qu'on a vu, euh, vous allez un peu } \\
\text { faire quelque chose, vous allez écrire. }\end{array}$ \\
\hline 476 & E. & Oh, oh. \\
\hline 477 & M. & Alors, écrire au brouillon, on mettra au propre après. \\
\hline 478 & E. & Oooh. \\
\hline 479 & M. & $\begin{array}{l}\text { Vous allez recopier ce petit, ce qu'il y a que vous jugez être la règle, alors deee, de la société des pay- } \\
\text { sans au Moyen Âge, la société féodale. Les paysans, que doivent-ils faire, le seigneur que doit-il faire ? } \\
\text { Euh, recopiez le morceau de phrase que vous trouvez être le bon. }\end{array}$ \\
\hline 480 & E. & Les morceaux de quoi? \\
\hline 481 & M. & Tu le cherches dans le petit morceau qu'on a lu, entre... ici, c'est écrit quelque part. \\
\hline 482 & E. & Mais de quoi ? \\
\hline 483 & M. & Tout ce que tu as commencé à lire, ce que toi tu as commencé à lire... \\
\hline 484 & E. & Je sais pas moi. \\
\hline 485 & E. & Qu'est-ce qu'on doit chercher? \\
\hline 486 & M. & Tu me copies la phrase ou des phrases. \\
\hline 487 & E. & D'où à où maître? \\
\hline 488 & M. & $\begin{array}{l}\text { Tu la trouves, trouve la phrase qui dit les règles, les règles qu'ils doivent respecter, oui, mais moi XX, } \\
\text { cherche, je sais que c'est pas expliqué, on va voir, cherche... dans le petit morceau. Bastien quoi dans } \\
\text { le STOP. Cherche! }\end{array}$ \\
\hline 489 & E. & Et on cherche où ? \\
\hline 490 & M. & $\begin{array}{l}\text { Des paysans. Ce que les paysans doivent à leur seigneur ce que les seigneurs doivent à leurs paysans... } \\
\text { Regarde, cherche... XX Allez, trouvez cette phrase ou les mots qui te semblent importants. Je veux } \\
\text { connaître les règles. }\end{array}$ \\
\hline
\end{tabular}


niques désuets aujourd'hui. Conscient de cette difficulté, mais dans l'impossibilité, comme dans un texte documentaire, d'indiquer immédiatement en apposition la définition du nouveau terme, le romancier, lorsque c'est possible, tente, parfois, de construire du sens autour des mots inhabituels dans l'espoir que la compréhension incomplète ne bloque pas l'évocation, ce qui n'est pas le cas en particulier dans cette séance.

Après une lecture silencieuse des deux premières pages, l'enseignant demande : «Qu'est-ce que vous reconnaissez ? ». Une élève cite le terme "serf » à bon escient, mais, très vite, la lecture de ces deux premières pages devient un obstacle à la compréhension, car elles contiennent un vocabulaire spécifique au monde religieux pourtant déjà étudié (en 119, « Rappelez-vous le plan de l'abbaye que vous avez sur le cahier d'histoire »). L'extrait ci-dessous est un comportant un lexique et le plan d'un monastère auquel il renvoie les élèves régulièrement, par exemple, en 139 : « Regarde dans ton vocabulaire». Il a cependant été dans l'obligation de faire du mot à mot. Vingt minutes ont été nécessaires pour arriver à comprendre les vingt-trois premières lignes à l'aide d'un déchiffrage laborieux du vocabulaire car les élèves ne possèdent pas « la compétence encyclopédique requise $»$ (Louichon, 2009, p. 12) et beaucoup décrochent d'autant que la compréhension d'un texte passant par l'interprétation correcte des marques linguistiques, ces mauvais lecteurs rencontrent beaucoup de difficultés dans la prise en compte des connecteurs ou des déterminants (Garcia-Debanc, 1991, p. 28). Mauvais lecteurs ou pas, certains des élèves de la classe, rebutés par cette « lecture-problème », restent en extériorité complète au texte et ils n'engagent aucune activité cognitive ou

\begin{tabular}{|l|l|l|}
\hline 136 & E. & L'office. \\
\hline 137 & M. & Y a ? \\
\hline 138 & E. & Y a un office. \\
\hline 139 & M. & Alors qu'est-ce que c'est un office ? Regarde dans ton vocabulaire. \\
\hline 140 & E. & Vocabulaire? \\
\hline 141 & M. & Qu'est-ce que c'est l'office hop regarde dessous. \\
\hline 142 & E. & Maître. \\
\hline 143 & M. & Qu'est-ce que c'est un office ? \\
\hline 144 & E. & C'est, c'est la partie de l'office, la prière qui se chante à l'aurore. \\
\hline 145 & M. & Alors qu'est-ce que c'est un office? \\
\hline 146 & E. & C'est pas celle-la. \\
\hline 147 & E. & C'est une partie qui se chante euh. \\
\hline 148 & M. & Tu es sûr là, regarde bien ton texte. \\
\hline 149 & E. & Qui se chante en aurore. \\
\hline 150 & M. & Non regarde ta phrase, c'est pas ça, qu'est-ce que c'est ? Loffice, c'est ? \\
\hline 151 & M. & Dis-le plus fort c'est? \\
\hline 152 & E. & C'est la prière. \\
\hline 153 & M. & $\begin{array}{l}\text { Donc, c'est la prière. Ce qui -, un office c'est la prière, on est dans un monastère, les gens prient } \\
\text { d'accord ? Qui c'est qui prie au fait ? }\end{array}$ \\
\hline
\end{tabular}

exemple de ce qui s'est produit de manière répétitive :

" Abbaye », "prieur », « chapelle», " chant des moines », « office des laudes », "porterie », « frère portier », " frère cellérier », " prieur » se succèdent et les élèves en ignorent le sens. L'enseignant avait pourtant prévu la difficulté en demandant d'ouvrir le livre d'histoire à la page consacrée à ce thème langagière. Décoder tous les mots d'un texte pour le comprendre n'est donc pas la clé de sa compréhension (Tauveron, 1999, p. 11). 
Limpact des personnages dans la compréhension de faits historiques

Les personnages peuvent affecter la compréhension d'une fiction historique pour la jeunesse de diverses façons à la fois sur le plan de l'identification des élèves aux héros et sur celui de l'historicité de ces personnages.

La catégorisation proposée par André Peyronie a été appliquée aux trois romans concernés par notre enquête, Sans nom, ni blason, Deux graines de cacao et Le faucon déniché. Si les œuvres proposées à la jeunesse n'offrent pas la même richesse de personnages que celle du roman d'Umberto Eco, nous pouvons supposer que les relations entretenues entre les personnages historiques et les personnages fictifs dotent ces derniers d'un genre d'historicité, une « sorte d'épaisseur historique imaginaire " variable selon les ouvrages. Examinons les personnages de ces trois romans.

Dans Deux graines de cacao d'Evelyne BrisouPellen (2001), les personnages historiques cités sont seulement au nombre de quatre, Christophe Colomb, Toussaint Louverture, Mozart et Bach et, en tant que personnages historiques-mentionnés, ils n'interviennent pas dans la fiction. La totalité des acteurs de ce récit sont donc des fictifs-actants, même si les esclaves, l'équipage, les familles de planteurs ont tous des modèles dans la réalité. Le lecteur est donc, par une action ne mettant en scène que des personnages inventés, plongé dans un imaginaire dont aucun personnage historique avéré ne peut l'en extraire et le ramener à la perception d'une réalité historique qui lui permettrait de se dire, en son for intérieur, que les faits présentés se sont réellement passés. Ce monde que l'auteur veut le plus près possible du réel par le soin apporté au moindre détail n'est que virtuel pour une majorité d'élèves négligeant nombre de précisions sur le cadre ou le contexte, car essentiellement centrés sur la quête d'identité du héros, quête en ellemême sans référents historiques concrets, d'autant que, dans ce cas précis, l'histoire de la traite négrière est souvent inconnue des élèves de cycle 3.

Dans le roman de Jean-Côme Noguès, Le faucon déniché, le seigneur Guilhem Arnal de Soupex est le seul personnage historique (connu uniquement sur le plan local) figurant dans cette fiction et comme il est très présent dans l'histoire, c'est donc un historique-actant. Tous les autres personnages dont le héros Martin sont inventés mais certains ont des modèles dans la réalité, correspondant à des entités historiques comme les paysans, les pèlerins. À la différence de Deux graines de cacao, la quasi-absence de personnages historiques ne semble pas toutefois un obstacle majeur car le cadre historique choisi, le temps des seigneurs et des paysans, est un des mieux connus des élèves de cycle 3 bercés depuis leur enfance par des récits mettant en scène cette société. Aussi l'auteur n'a-t-il pas eu besoin d'introduire davantage de personnages historiques pour assurer l'historicité de son roman.

Une des classes - un double niveau CM1/CM2 concernées par la lecture de ce roman, a lu en séance de français, avant que la séquence d'histoire ne débute, les deux premiers chapitres, «Le nid » et "Le fauconnier », puis l'enseignant a alterné systématiquement séances de français et séances d'histoire. À la suite de la lecture des chapitres 7 (lecture silencieuse) et 8 (lecture à haute voix), «Guilhem de Soupex » et "Traqué », une première série de questionnaires a été soumise aux élèves portant sur la dichotomie fiction / réalité. À la question 8 , « quels sont d'après toi, les personnages qui peuvent avoir réellement existé ? », excepté un seul élève pour lequel aucun n'avait pu exister et un autre qui n'a rien répondu, la plupart ont validé l'existence des gardes, des seigneurs, des chapelains, des paysans, du fauconnier, du faucon, de Guilhem et de sa femme (« car les seigneurs avaient des femmes! » écrit un élève) et de Martin. Ce dernier n'est cependant cité que six fois sur vingt et une réponses et, souvent, après coup. Si les élèves valident plus facilement les catégories dont ils ont déjà souvent entendu parler par différents biais, il leur semble moins évident, apparemment, que Martin ait réellement existé car si les gardes, les seigneurs, les paysans réfèrent à des modèles historiquement attestés, il leur est plus difficile de justifier de l'existence du jeune serf car c'est le héros à qui il arrive des aventures extraordinaires : il sauve le château d'une attaque, il défie son seigneur pour un faucon. Il ne peut être un enfant « ordinaire $» . .$.

Le roman Sans nom ni blason offre en partie la même configuration quant aux personnages. L'histoire se déroule au Moyen Âge et les allusions aux mondes des seigneurs et des paysans y abondent. Le personnage principal et les personnages secondaires y sont tous fictifs: Guillaume, Arnaud de Craon, le comte Bérard, Bertrand, Jean-le-rat. Dans la première moitié de la fiction apparaissent deux 
historiques mentionnés (Aristote et Platon, p. 61) et quatre historiques rattachés (le pape, le roi de Léon, le roi de France Louis VII et son épouse Aliénor d'Aquitaine, p. 9, 48). Or, à partir du moment où l'écrivain ancre l'histoire de Guillaume dans celle des croisades et évoque avec détails des contextes historiques très précis, il cite de nombreuses grandes figures de cette épopée (p. 87-89, 124, 158, 169, 171, 177) inconnues des élèves, mais crédibilisant ainsi l'histoire romanesque.

Point commun à ces deux dernières fictions et, du reste, à l'ensemble des romans historiques, que certains de leurs personnages soient historiques n'empêchent pas nos deux auteurs de leur prêter des propos totalement imaginaires comme cela a été déjà souligné.

Ces trois romans offrent cependant une similitude présente dans la majorité des fictions histo- riques pour la jeunesse. Ils suivent le destin d'un enfant ou d'un adolescent facteur de l'adhésion des lecteurs à l'histoire racontée. Ainsi, d'après Brigitte Louichon, le roman d'Evelyne Brisou-Pellen est un roman de l'enfant trouvé et, à ses yeux, « lire Deux graines de cacao pour un enfant, c'est embarquer dans une aventure qui est moins maritime et historique qu'intérieure "(Louichon, 2014, p. 181). Le lecteur souhaite avant tout savoir qui est Julien, quels sont ses parents et dans cette perspective, la question du contexte historique serait secondaire. Ce qui importe, c'est l'engagement du sujet lecteur.

C'est ce qui a pu être constaté lors de la séance consacrée à la lecture du premier chapitre de Sans nom ni blason. À un moment donné, tous les élèves ont manifesté un regain d'intérêt, notable lorsque leur maître les a amenés à s'interroger sur le personnage principal :

\begin{tabular}{|l|l|l|}
\hline 339 & M. & Qui c'est Guillaume ? \\
\hline 340 & E. & C'est le petit enfant. \\
\hline 341 & E. & Le bébé abandonné. [...] \\
\hline 346 & M. & Non mais attends, qui est Guillaume? [...] \\
\hline 354 & E. & Le petit enfant. \\
\hline 355 & M. & Y a un petit enfant ou non ? \\
\hline 356 & E. & Le grand mince. \\
\hline 357 & M. & Ah ! Le ? \\
\hline 358 & E. & Le grand mince. \\
\hline 359 & M. & $\begin{array}{l}\text { Le grand mince. Regarde le début, que faisait ici Guillaume à cette heure ? Y a que trois personnages : } \\
\text { Arnaud de Craon, le frère portier, le grand mince. }\end{array}$ \\
\hline 360 & E. & Eh c'est le grand mince leeu... celui qu'on avait abandonné. \\
\hline 361 & M. & Puisque ? Alors le grand mince c'est ? \\
\hline 362 & EE. & Le bébé abandonné. Guillaume ! \\
\hline 363 & M. & Alors quand c'est qu'il avait, que i peut être bébé et grand mince à la fois ? \\
\hline 364 & EE. & Non, non, après \\
\hline 365 & E. & C'est parce qu'il a changé de euh... \\
\hline 366 & E. & 18 ans. \\
\hline 367 & E. & Il a 18 ans qui, qu'il est abandonné, qu'il est abandonné. \\
\hline 368 & M. & Donc, on est, Théo ? \\
\hline 369 & E. & 18 ans plus tard. \\
\hline 370 & M. & $\begin{array}{l}18 \text { ans plus tard, comme dans le cinéma, il a fait un petit retour en arrière, il y a dix-huit ans, il l'avait } \\
\text { trouvé, euh au fait où il l'avait trouvé ? }\end{array}$ \\
\hline
\end{tabular}


Les élèves découvrent un des éléments clés. Ils comprennent tout à coup que le grand mince était Guillaume et que ce jeune garçon n'était autre que le bébé déposé dix-huit ans plus tôt. L'enthousiasme dans la classe a été palpable à ce moment-là, tous les élèves se sentant concernés par l'histoire de Guillaume, phénomène illustrant nettement l'importance de la fonction implicative dans l'attention des élèves (Reuter, 2007), mais peu bénéfique à la construction de savoirs historiques scolaires. Même s'il est difficile d'associer la notion de "personnage préféré » à celle d' "identification », la question 6 du questionnaire évoqué plus haut, relatif au roman Le faucon déniché, souligne un point intéressant. Elle demandait aux élèves : «Quel est ton personnage préféré ? Pourquoi ?». Or, le héros principal, Martin, n'est pas toujours choisi. Sur vingt-trois élèves présents, dix-sept ont cependant opté pour lui et quatorze ont justifié leur réponse : il est courageux (4), il sauve le château, le village, tout le monde (3), il s'est évadé (2), il est marrant (1) mais aussi parce que simplement à leurs yeux, c'est le héros, le personnage principal (4). Cinq élèves lui ont toutefois préféré le seigneur Guilhem Arnal de Soupex pour des raisons différentes mais convergentes : en tant que seigneur, il est très fort, vaillant, c'est un guerrier, c'est le roi. Un des élèves dit clairement qu'il souhaiterait être à sa place. Deux élèves ont choisi le faucon. Un seul justifie sa réponse : il aime les animaux. Penser le passé par analogie ramène chacun à ses préférences...

\section{Souvenirs de lecture}

Une autre expérience a permis d'observer un cas de figure différent. Alors que des élèves de CMl ont lu au préalable et avec intérêt le roman Deux graines de cacao, leur enseignant a voulu par la suite amener ses élèves de la fiction à la réalité historique à partir de l'étude de sources de nature variée témoignant des terribles sanctions infligées aux esclaves qui tentaient de s'enfuir ou qui désobéissaient. Or, le réinvestissement de cette lecture dans une perspective historique a été problématique alors que l'on aurait pu penser le contraire. Quelques exemples seront présentés.

Ainsi, un des documents historiques, un extrait du Code noir, choisi par le professeur des écoles, illustrait parfaitement un passage du roman où des scènes de tortures sont dépeintes avec une extrême précision en lien, justement, avec le Code noir, cité nommément dans le texte littéraire :

Extrait du roman Deux graines de cacao, p. 231-232: « il y avait ce qu'on appelait le « Code noir » et, normalement, on ne pouvait pas torturer. Si l'esclave s'enfuyait, on pouvait quand même lui couper les oreilles, la première fois, lui trancher le jarret la deuxième, le tuer la troisième...».

Lors de la séance d'histoire à visée d'éducation aux droits de l'homme qui a suivi la séquence de littérature, le maître a fait lire les articles du Code noir par les élèves pensant qu'ils feraient le lien :

Article du Code noir lu par un élève (Nathanaël) : "L'esclave fugitif qui aura été en fuite pendant un mois, à compter du jour que son maître l'aura dénoncé en justice, aura une oreille coupée et sera marqué au fer rouge d'une fleur de lys à une épaule. S'il recommence à un autre mois, il aura le jarret coupé et il sera marqué d'une fleur de lys sur l'autre épaule, et, la troisième fois, il sera puni de mort ».

Or, les hypothèses présentées par les élèves l'ont été uniquement en relation avec leur vécu, par analogie, ou en référence à leurs savoirs sociaux, et aucun n'a pensé à mettre en relation l'extrait du roman concerné et l'article du Code noir alors qu'ils étaient en adéquation totale. Ce qui est d'autant plus étonnant que ce passage est un moment fort de la prise de conscience opérée par le héros, Julien, et qu'il avait suscité, lors de sa lecture, de vives réactions de la part de la classe.

Deux exemples de mise en relation ont cependant pu être observés. 
Dès le début de la séance, le maître demande aux élèves ce qu'ils se rappellent :

\begin{tabular}{|l|l|l|}
\hline 1 & M. & De quoi te rappelles-tu ? \\
\hline 2 & E. & Y a des médecins, (plus fort) y a des médecins. \\
\hline 3 & M. & Pardon ? \\
\hline 4 & E. & Y a des médecins. \\
\hline 5 & M. & Des médecins. \\
\hline 6 & E. & Y a soi-disant médecins dans les bateaux. \\
\hline 7 & M. & Oui. \\
\hline 8 & E. & Oui pour savoir si s'ils étaient en bonne santé, ils devaient décrire des parties. \\
\hline 9 & M. & $\begin{array}{l}\text { Du corps, on avait vu ça, mais bon, oui, tu as retenu ça, pourquoi pas ? Donc ça c'était pas...., } \\
\text { c'était... Où est-ce qu'on avait vu ça s'il te plaît, Julien? }\end{array}$ \\
\hline 10 & Julien & Euh dans le livre Deux graines de cacao ? \\
\hline
\end{tabular}

Un des élèves, à la question du maître relative à la traite négrière s'est souvenu, en apportant des détails qu'il ne pouvait tirer que de la lecture de Deux graines de cacao, de l'examen médical minutieux subi par les esclaves avant leur embarquement, examen opéré par Gabriel, le jeune séminariste, compagnon fidèle de Julien, tout au long dans son expédition.
Or, dans la seconde partie de la séance, alors que le maître fait étudier l'extrait d'un ouvrage datant de 1834, Voyage pittoresque historique au Brésil écrit par Jean-Baptiste Debray, extrait dépeignant les punitions infligées dans les plantations aux esclaves fugitifs, un élève s'interroge sur le sort réservé aux corps de ceux qui périssaient à la suite de ces mauvais traitements :

\begin{tabular}{|l|l|l|}
\hline 508 & E. & Quand ils meurent, ils les enterrent ou ils les brûlent ? \\
\hline 509 & M. & Qui ? autre élève propose une réponse, en apparence, sans lien avec le contexte examiné. \\
\hline 510 & E. & Mais non, ils les jettent, ils les jettent à l'eau... \\
\hline 511 & M. & $\begin{array}{l}\text { Ce que confirme le maître : } \\
\text { Alors, si tu es sur un bateau qui t'emmène d'Afrique... sur ton lieu d'esclavage de l'autre côté de } \\
\text { l'Atlantique, on s'embête pas, on te détache, on te jette à l'eau. }\end{array}$ \\
\hline 512 & E. & $\begin{array}{l}\text { Et celui qui avait répondu, ponctue : } \\
\text { Voilà ! }\end{array}$ \\
\hline
\end{tabular}

« Chirurgien. Il était chirurgien. Son rôle était de choisir. Il devait examiner l'allure, la peau, les organes... Il devait aussi les faire marcher et courir, étendre les bras et jambes, tousser violemment [...] et même leur lécher plusieurs parties du corps, pour détecter au goût de la sueur les maladies », extrait de E. Brisou-Pellen, Deux graines de cacao, Hachette Jeunesse, 2001, p. 120.

Dans ce premier cas, les souvenirs de lecture de l'élève lui ont apporté des éléments de réponse, des connaissances sur un point précis de la traite négrière (la sélection des esclaves avant l'embarquement). Ils témoignent d'une interprétation adaptée au contexte historique de la question posée par le maître.
Apparemment la mémoire de cet élève a été marquée par les images fortes d'un passage du roman d'Evelyne Brisou-Pellen dans lequel le capitaine donne l'ordre de jeter à la mer les esclaves morts de la variole. Ses souvenirs de lecture traduisent, cependant, une compréhension erronée de la scène évoquée par la source historique. Les esclaves concernés vivent sur terre, dans des plantations souvent éloignées de la mer, ils ne sont pas sur un bateau. La question de l'élève s'inquiétant du sort réservé aux corps des esclaves morts à la suite de tortures interpelle, toutefois, la subjectivité de cet autre élève, qui, certainement touché par l'épisode cruel du roman historique, réactive et projette un souvenir inadapté à la situation mais seul valide à ses yeux. Puis conforté 
par la réponse du maître, il peut asséner un « voilà » significatif. Mais se souvenir de ce qu'on a lu et compris, condition nécessaire à la constitution d'une culture (Louichon, 2009, p. 160), s'accompagne en histoire, mais également dans toutes les autres disciplines, de la capacité à mobiliser ses souvenirs à bon escient afin de construire une culture partagée. Or cet élève, sujet-lecteur, focalisé par ses souvenirs de lecture, reste dans son monde dans lequel le maître l'a, un moment, rejoint. En effet, ce dernier semble s'être, intuitivement, souvenu du passage de Deux graines de cacao, puisque, dans sa reprise, il prend en compte ce souvenir de lecture partagé, mais l'absence d'explications peut être dommageable pour les autres élèves dont la mémoire n’a pas forcément stocké les mêmes souvenirs.

Utiliser un roman historique en histoire pose nécessairement la question de la compréhension du récit, ce qui conduit à prendre en compte des facteurs individuels et des facteurs textuels. Or, le processus interprétatif est d'autant plus difficile qu'il se fonde sur des indéterminations inhérentes au texte, appelées parfois « lieux d'incertitude ${ }^{205}$ » et que le roman préférant « toujours l'événement au tableau, c'est-à-dire la représentation au discours explicite $[\ldots]$, c'est en montrant qu'il donne à penser » (Jouve, 2004, p. 107). Comme le souligne Vincent Jouve, « dégager la signification d'un roman, c'est donc obtenir du conceptuel à partir de l'événementiel. Une telle opération peut difficilement échapper à la subjectivité » (id., p. 108), chaque lecteur créant «sa » fiction à partir de ses perceptions sélectives. S'il faut, toutefois, tenir compte du fait que la dimension subjective et la question de la mémoire du lecteur sont différentes dans un contexte de lecture privée et dans un contexte scolaire en lecture suivie, il est nécessaire, cependant, de se demander quelle " histoire » construisent les histoires lues par des élèves, sujets-lecteurs singuliers, chacun fictionnalisant l'œuvre à sa manière (Langlade, 2006 ; 2009 , p. 47), en investissant, en complétant ou en détournant les espaces fictionnels qu'elle lui offre, le sens étant reconfiguré par le contexte scolaire. En outre, exploiter un roman historique en classe prend du temps. Plusieurs séances sont nécessaires et, au fur et à mesure de la lecture, se construit, chapitre par chapitre, une mémoire de l'histoire, au sens de fabula, sélective et partiale, donc partielle et insuffisante car l'élève ne réussit pas toujours à transposer ses souvenirs de lecture à un thème historique simi- laire mais abordé différemment. Se pose également la question du déroulé des séquences. Si on se place du point de vue de l'historien, doit-on d'abord étudier l'œuvre en français puis ensuite en histoire (solution qui a la préférence de Brigitte Louichon (2014, p. 185-186) ? Vaut-il mieux l'étudier en alternant les deux disciplines? Dans les deux cas, il semble que, dans une perspective d'acquisition de savoirs historiques scolaires, toute exploitation de roman historique à l'école devrait amener les élèves à constater ce qui les a conduits à projeter dans le texte ce qui n'y était pas, cette auto distanciation (Jouve, 2004, p. 110) rejoignant une des finalités de l'histoire qui est de s'approcher au plus près du vrai et de développer l'esprit critique.

\section{NOTES}

1. Son usage en classe est déjà régulièrement questionnée : entre autres, Cariou, 2012b ; Jaubert, Lalagüe-Dulac et Louichon, 2013 ; Louichon, 2014 ; Dumortier, Granata, Raxhon et Van Beveren, 2015.

2. Pour une typologie complète, voir Peltier, 2008, p. 10-17; Solet, 2003.

3. Par exemple, Philippe Barbeau à propos de l'écriture du roman, La guerre d'Eliane :[http://philippe.barbeau.pagesperso-orange.fr/bibliographie/documents\%20GE.htm].

4. L'ensemble du cadre théorique de la didactique de l'histoire ne peut être abordé ici dans sa totalité. Aussi, développerons-nous ce qui semble éclairer plus particulièrement notre problématique. Nous ne rappellerons pas, par exemple, certains points déjà développés avec clarté par Didier Cariou (2012b) quant aux relations entre l'histoire (en tant que discipline) et la littérature, objet de nombreux débats sur le plan épistémologique et historiographique. Pour une définition actualisée de la didactique de l'histoire et ses questionnements, voir Doussot, 2011 ; Cariou, 2012a; Lalagüe-Dulac, Legris et Mercier, 2016.

5. Pour une définition, Launay (2004, p. 55), pour lequel la notion de représentation désigne "le processus de mise en correspondance entre deux éléments qui aboutit à ce que l'un (le représentant) répète, remplace ou "présente autrement" l'autre (le représenté) ».

6. Moscovici, 1961 ; Moscovici, 1984 ; Jodelet, 1984 ; Cohen-Azria, 2007.

7. Giordan et De Vecchi (1987) : le terme de « représentation » implique trop souvent l'idée d'une représentation graphique des objets, source possible de confusion. 
8. Pour Giordan et De Vecchi (1987, p. 82 et p. 85), « les conceptions peuvent évoluer, à travers les étapes du développement mental, vers une compréhension plus poussée, ce qui se traduit souvent, soit par une complexification de l'argumentation, soit par un changement de préoccupations ».

9. Bayard (1998, p. 112) cité par Langlade (2004, p. 85). Jouve (2004, p. 105) : « la lecture d'un texte étant toujours lecture du sujet par lui-même ». Louichon (2009, p. 140-141) : « le souvenir de lecture est par nature un souvenir de soi $»$.

10. Sur la confusion fiction / littérature et une mise au point, voir Dumortier (2001).

11. Barbey d'Aurevilly, dans sa préface à la seconde édition de L'Ensorcelée, dans Euvres romanesques complètes, éd. de J. Petit, Pléiade, 1964, t. 1, p. 1351, cité par Ansel (2000, p. 110).

12. Bayard, 1998, p. 130.

13. Implication renforcée par «l'effet de vie » comme «le choix de patronymes courants, moyen constant de favoriser la confusion entre le monde fictionnel et le ME » (Dumortier, 2001).

14. Également présente dans la lecture littéraire définie comme un va-et-vient entre participation et distanciation (Dufays, Gemenne \& Ledur, 2005, p. 96).

15. Ainsi que Mazauric, Fourtanier et Langlade (2011) le rappellent, " la notion de "texte du lecteur" apparaît en fait, sous une forme ou sous une autre, dans toutes les approches de la lecture littéraire qui accordent une place déterminante à l'activité des lecteurs dans l'actualisation et la reconfiguration des œuvres » (p. 20). Pour une définition du texte de lecteur en situation scolaire, voir également Dufays (2011).

16. Sept sont dénombrées : véhiculaire, structurante, objectivante, médiatrice, implicative, intégrative et, enfin, transitionnelle (Reuter, 2007, p. 9-10).

17. Onze séances, quarante-huit travaux d'élèves, plus de deux cents cinquante réponses de Professeur des écoles, stagiaires ou titulaires, à un même questionnaire, une dizaine d'écrits produits lors d'animations pédagogiques, quinze mémoires professionnels, des travaux menés sur des corpus de fictions historiques spécifiques, constituent un corpus qui sera partiellement pris en compte.

18. Lalagüe-Dulac, 2012.

19. Élaborés avec l'aide de Maryse Rebière.

20. Dufays, 1994, p. 156-157, qui fait de l'ambiguité, du résidu, du blanc et de la contradiction des « lieux d'incertitude », autant d'interstices où s'engouffre la subjectivité du lecteur.

\section{RÉFÉRENCES}

Ansel, Y. (2000). Lirrésistible ascension du romanesque dans le roman historique. Dans D. Peyrache-Leborgne $\&$ D. Couégnas (dir.), Le roman historique. Récit et histoire (p. 110-117). Nantes, France : Éditions Pleins Feux.

Baguley, D. (2008). Histoire et fiction. Les RougonMacquart de Zola. Dans C. Reffait, (dir.), Romanesque et histoire (p. 68-82). Amiens, France : Centre d'Études du Roman et du Romanesque de l'université de Picardie - Jules Verne.

Bayard, P. (1998). Qui a tué Roger Ackroyd ? Paris : Éditions de Minuit.

Bernié, J.-P. (2002). L'approche des pratiques langagières scolaires à travers la notion de " communauté discursive » : un apport à la didactique comparée ? Revue française de pédagogie, 141, 77-88.

Bernié, J.-P., Jaubert, M., \& Rebière, M. (2004). L'hypothèse « communauté discursive ». Les cahiers Théodile, 4, 51-80.

Bessière, J., \& Sinoploi, F. (dir.) (2005). Storia e memoria nelle riletture e riscritture letterarie / Histoire, mémoire et relectures et réécritures littéraires. Rome, Italie : Bulzoni Editore.

Bordas, E. (2002). De l'historicisation des discours romanesques. Revue d'histoire du xixè siècle, 25, 171-197.

Brisou-Pellen, E. (2001). Deux graines de cacao. Paris, France : Hachette Jeunesse.

Bruno, P., \& Geneste, P. (2008). Le Roman pour la jeunesse. Dans D. Escarpit (dir.), La Littérature de jeunesse. Itinéraires d'hier à aujourd'hui (p. 390-446). Bordeaux, France : Éditions Magnard.

Cariou, D. (2003a). Le raisonnement par analogie, un outil au service de la construction du savoir en histoire par les élèves (Thèse de doctorat, université de Picardie JulesVerne, Lille, France).

Cariou, D. (2003b). Représentations sociales et didactique de l'histoire. Le cartable de Clio, 3, 169-178.

Cariou, D. (2004). La conceptualisation en histoire au Lycée : une approche par la mobilisation et le contrôle de la pensée sociale des élèves. Revue Française de Pédagogie, 147, 57-67

Cariou, D. (2006). Le contrôle de la pensée naturelle en situation didactique. Dans V. Haas (éd.), Les savoirs du quotidien. Transmissions, appropriations, représentations (p. 119-130). Rennes, France : Presses universitaires de Rennes.

Cariou, D. (2012a). Écrire l'histoire scolaire. Quand les élèves écrivent en classe pour apprendre l'histoire. Rennes, France : Presses universitaires de Rennes.

Cariou, D. (2012b). Littérature de jeunesse et enseignement de l'histoire au cycle 3. Repères, 45, 163-179.

Clément, P. (1994). Représentations, conceptions, connaissances. Dans A. Giordan, Y. Girault \& P. Clément (éd.), Conceptions et connaissances (p. 15-45). Berne, Suisse : Peter Lang. 
Cohen-Azria, C. (2007). Représentations. Dans Y. Reuter (dir.), Dictionnaire des concepts fondamentaux des didactiques (p. 197-202). Bruxelles, Belgique : De Boeck.

Deleplace, M. (2006). Les apprentissages conceptuels en histoire. La "révolution" entre sens commun et sens scolaire. Dans V. Haas (éd.), Les savoirs du quotidien. Transmissions, appropriations, représentations (p. 91-104). Rennes, France: Presses universitaires de Rennes.

Dortier, J.-F. (dir.) (2004). Le dictionnaire des sciences humaines. Auxerre, France : Éditions Sciences Humaines.

Doussot, S. (2011). Didactique de l'histoire. Outils et pratiques de l'enquête historienne en classe. Rennes, France: Presses universitaires de Rennes.

Dufays, J.-L. (1994). Stéréotype et lecture. Essai sur la réception littéraire. Liège, Belgique : Mardaga.

Dufays, J.-L. (2011). Les textes du lecteur en situation scolaire. Dans C. Mazauric, M.-J. Fourtanier \& G. Langlade (dir.), Le texte du lecteur, vol.3 (p. 19-32). Bruxelles, Belgique : Éditions Peter Lang.

Dufays, J.-L. (2013). Sujet lecteur et lecture littéraire : quelles modélisations pour quels enjeux ? Recherches E Travaux, 83, 77-88.

Dufays, J.-L., Gemenne, L., \& Ledur, D. (2005). Pour une lecture littéraire. Bruxelles, Belgique : Éditions De Boeck Supérieur.

Dumortier, J.-L. (2001). Lire le récit de fiction. Pour étayer un apprentissage : théorie et pratique. Bruxelles, Belgique : De Boeck-Duculot.

Dumortier, J.-L., Dispy, M., \& Van Beveren, J. (2011). Pour le discours incitatif. Repères, 43, 31-52.

Dumortier, J.-L., Granata, V., Raxhon, Ph., \& Van Beveren, J. (2015). Devoir de mémoire et pouvoir des fictions. Namur, Belgique : Presses universitaires de Namur.

Eco, U. (1985). Apostille au "Nom de la rose». Paris : Grasset, Le Livre de Poche.

Garcia-Debanc, C. (1991). Lire le Moyen Âge ou quels critères pour différencier roman historique et écrit d'historien. Pratiques, 69, 7-42.

Gengembre, G. (2006). Le roman historique. Paris, France : Klincksieck.

Giordan, A., \& Vecchi (de), G. (1987). Les origines du savoir. Des conceptions des apprenants aux concepts scientifiques. Paris, France: Delachaux et Niestlé.

Goigoux, R., \& Cèbe, S. (2011). Comprendre et mémoriser les récits à l'école : se souvenir de ce que le texte ne dit pas. Dans G. Toupiol (dir.), Mémoire, langage et apprentissage (p. 33-52). Paris, France : Éditions Retz.

Heimberg, C. (2005). La comparaison et l'ouverture à l'autre pour donner du sens à l'histoire enseignée. Le Cartable de Clio, 5, 44-55.

Jaubert, M., Lalagüe-Dulac, S., \& Louichon, B. (2013). Les fictions historiques : un objet littéraire, éditorial et scolaire qui interroge les frontières. Repères. Recherches en didactique du français langue maternelle, 48.

Jodelet, D. (1984). Représentations sociales : phénomènes, concepts et théorie. Dans S. Moscovici, Psychologie sociale (p. 357-378). Paris, France : Presses universitaires de France.

Jouve, V. (2004). La lecture comme retour sur soi : de l'intérêt pédagogique des lectures subjectives. Dans A. Rouxel \& G. Langlade (dir.), Le sujet lecteur. Lecture subjective et enseignement de la littérature (p. 105-114). Rennes, France : Presses universitaires de Rennes.

Jouve, V. (2008). La Lecture. Paris, France: Hachette.

Lalagüe-Dulac, S. (2012). Le rôle du langage dans la construction de savoirs historiques sensibles et de compétence éthiques. Spiral-E, Revue de recherches en éducation. Supplément électronique, 49, 19-32.

Lalagüe-Dulac, S., Legris, P., \& Mercier, C. (2016). Didactique et histoire. Des synergies complexes. Rennes, France: Presses universitaires de Rennes.

Langlade, G. (2004). Le sujet lecteur auteur de la singularité de l'œuvre. Dans A. Rouxel \& G. Langlade (dir.). Le sujet lecteur. Lecture subjective et enseignement de la littérature (p. 81-92). Rennes, France : Presses universitaires de Rennes.

Langlade, G. (2006). Lactivité "fictionnalisante" du lecteur. Dans M. Braud, B. Laville \& B. Louichon (dir.), Les enseignements de la fiction (p. 163-176). Bordeaux, France : Presses universitaires de Bordeaux.

Langlade, G. (2009). Activité fictionnalisante du lecteur et dispositif de l'imaginaire. Dans M. Roy, M. Brault \& S. Brehm (dir.), Formation des lecteurs. Formation de l'imaginaire (p. 46-65), Cahiers Figura, n²0.

Launay, M. (2004). Psychologie cognitive. Paris, France : Hachette supérieur.

Lautier, N. (1997). À la rencontre de l'histoire. Villeneuve d'Ascq, France : Presses universitaires du Septentrion.

Lautier, N. (2001). Les enjeux de l'apprentissage de l'histoire. Perspectives documentaires en éducation, 53, 61-68.

Lautier, N., \& Allieu-Mary, N. (2008). La didactique de l'histoire. Revue française de pédagogie, 162, 95-131.

Louichon, B. (2009). La littérature après coup. Rennes, France: Presses universitaires de Rennes.

Louichon, B. (2014). Lecture du roman historique. Lexemple de Deux graines de cacao. Dans J. Van Beveren (dir.), Littérature, langue et didactique (p. 173-187). Namur, Belgique : Presses universitaires de Namur.

Manson, M. (2013). Roman historique. Dans I. NièresChevrel \& J. Perrot (dir.), Dictionnaire du livre de jeunesse en France (p. 822-826). Paris, France : ÉlectreÉditions du Cercle de la Librairie.

Mazauric, C., Fourtanier, M.-J., \& Langlade, G. (2011). Présentation. Dans C. Mazauric, M.-J. Fourtanier \& G. Langlade (dir.), Le texte du lecteur, vol. 2, (p. 19-25). Bruxelles, Belgique : Éditions Peter Lang.

Mirande, J. (1997). Sans nom ni blason. Paris, France : Pocket Jeunesse.

Moniot, H. (1993). Didactique de l'histoire. Paris, France: Nathan.

Moscovici, S. (1961). La psychanalyse, son image et son public. Paris, France: Presses universitaires de France. 
Moscovici, S. (1984). Introduction, le domaine de la psychologie sociale. Dans S. Moscovici (dir.), Psychologie sociale (p. 5-20). Paris, France : Presses universitaires de France.

Noguès, J.-C. (1972). Le faucon déniché. Paris, France : Le livre de Poche.

Peltier, M. (2008). Lire des romans historiques au quotidien, cycle 3. Dijon, France: CRDP Bourgogne.

Peyronie, A. (2000). Note sur une définition du roman historique suivie d'une excursion dans Le Nom de la rose. Dans D. Peyrache-Leborgne \& D. Couegnas (dir.), Le roman historique : récit et histoire (p. 278-289). Nantes, France : Pleins Feux.

Picard, M. (1986). La lecture comme jeu, Essai sur la littérature. Paris, France : Éditions de Minuit.

Prost, A. (1996). Histoires, vérités, méthodes. Des structures argumentatives de l'histoire. Le Débat, 92, 127-140.

Reuter, Y. (2007). Récits et disciplines scolaires. Présentation du numéro. Pratiques, 133/134, 9-10.
Solet, B. (2003). Le roman historique : invention ou vérité. Paris, France : Éditions du Sorbier.

Tauveron, C. (1999). Comprendre et interpréter le littéraire à l'école : du texte réticent au texte proliférant. Repères, 19, 9-38.

Tutiaux-Guillon, N. (1998). L'enseignement et la compréhension de l'histoire sociale au collège et au lycée. L'exemple de la société d'Ancien Régime et de la société du XIX ${ }^{e}$ siècle (Thèse à la carte, Villeneuve-d'Ascq, France : Presses du Septentrion).

Tutiaux-Guillon, N. (2009). L'histoire scolaire au risque des sociétés en mutation. La Revue française d'éducation comparée, 4(1), 11-15.

Vibert, A. (2013). Faire place au sujet lecteur en classe: quelles voies pour renouveler les approches de la lecture analytique au collège et au lycée? Eduscol : Ressources pour le collège et le lycée. Repéré à : [http://eduscol. education.fr/] 